\title{
El emprendimiento como cultura: ¿una estrategia para potenciar las competencias ciudadanas y el trabajo en grupo en la primera infancia?*
}

\author{
Entrepreneurship as a Culture: \\ A Strategy to Strengthen Citizenship Skills and Teamwork in Early Childhood?
}

\author{
Adriana María Gallego Henao', Verónica Torres Pabón², Estefanía Becerra Sierra3, \\ Katherine Córdoba Pérez ${ }^{4}$, Paula Andrea Pérez Castaño ${ }^{5}$
}

Para citar este artículo: Gallego, A. M., Torres, V., Becerra, E. Córdoba, K. Pérez, P. A. (2018). El emprendimiento como cultura: ¿una estrategia para potenciar las competencias ciudadanas y el trabajo en grupo en la primera infancia? Infancias Imágenes, 17(2), 176-184

\section{Resumen}

En este artículo se muestran los resultados de investigación sobre la cultura del emprendimiento como herramienta para desarrollar las competencias ciudadanas y el trabajo en equipo en los niños de nivel jardín de un centro educativo de la ciudad de MedeIlín, Colombia. Para ello, se acudió al paradigma cualitativo con un enfoque hermenéutico, privilegiando como estrategia de investigación el estudio de caso. Los participantes fueron cuatro maestras titulares de los niveles pre-jardín y jardín; se aplicaron técnicas de recolección como el grupo focal, observación no participante, talleres lúdicos y entrevista semi-estructurada. Se encontró que para potenciar habilidades tales como el trabajo en equipo y la creatividad a través de la cultura del emprendimiento es necesario
Recibido: 21-febrero-2018 / Aprobado: 23-julio-2018

que los docentes partan de las necesidades e intereses de sus estudiantes. Se concluye que la cultura del emprendimiento es una oportunidad para potenciar habilidades y competencias ciudadanas enfocadas al desarrollo integral de los niños.

Palabras clave: cultura, creatividad, enseñanza en equipo, maestro.

\begin{abstract}
This article shows the results of research on the culture of entrepreneurship as a tool to develop citizen competencies and teamwork in kindergarten-level children of an educational center in the city of Medellin, Colombia. To this end, a qualitative paradigm was used with a hermeneutic approach, favoring the case study as a research strategy. Participants were
\end{abstract}

* Este artículo es derivado de la investigación "Cultura del emprendimiento en el contexto escolar". Iniciada en julio de 2015 y finalizada en junio de 2016.

1 Licenciada en Educación Preescolar; Especialista en Docencia Investigativa Universitaria; Magister en Educación y Desarrollo Humano. Coordinadora de la Maestría en Educación y docente investigadora, Facultad de Educación y Humanidades, Escuela de Posgrados, Universidad Católica Luis Amigó-Funlam. Correo electrónico: adriana.gallegohe@amigo.edu.co

2 Licenciada en Educación Preescolar, Facultad de Educación y Humanidades, Universidad Católica Luis Amigó-Funlam. Correo: vero9430@hotmail.es

3 Licenciada en Educación Preescolar, Facultad de Educación y Humanidades, Universidad Católica Luis Amigó-Funlam. Correo: estefania-ve@hotmail.com

$4 \quad$ Licenciada en Educación Preescolar, Facultad de Educación y Humanidades, Universidad Católica Luis Amigó-Funlam. Correo: katecordoba@hotmail.com

5 Licenciada en Educación Preescolar, Facultad de Educación y Humanidades, Universidad Católica Luis Amigó-Funlam. Correo: paulaandrea_832@hotmail.com 
four tenured teachers from pre-kindergarten and kindergarten levels. Collection techniques—such as focus group, non-participant observation, play workshops and semi-structured interview - were applied. It was found that in order to enhance skills such as teamwork and creativity through the entrepreneurship culture, it was necessary for teachers to start from the needs and interests of their students. It is concluded that the culture of entrepreneurship is an opportunity to empower citizen skills and competencies focused on the integral development of children.

Keywords: culture, creativity, team teaching, teacher.

\section{Introducción}

El problema que se detectó en este contexto se dio a partir de algunas conversaciones informales con las maestras de la institución escenario de este estudio así como de un análisis a los documentos legales como el plan educativo institucional (PEI) y proyectos de aula, sobre la fundamentación conceptual que tienen las maestras de esta institución educativa (IE). Es decir, se sabe que ellas abordan los proyectos de emprendimiento, pero surge la necesidad de saber si ellas comprenden a qué se refiere este término, cuál es su fundamentación, cuál es la finalidad de enseñar el emprendimiento como cultura desde la primera infancia y a qué objetivos le apuesta el Ministerio de Educación Nacional (MEN) al insistir que esta área se debe enseñar en todas las IE.

Al revisar la literatura existente con relación al tema se encontró que la Ley 1014 del 2006 menciona que la formación para el emprendimiento propende por el

[...] desarrollo de la cultura del emprendimiento con acciones que buscan entre otros la formación en competencias básicas, competencias laborales, competencias ciudadanas y competencias empresariales dentro del sistema educativo formal y no formal y su articulación con el sector productivo. (Congreso de la República de Colombia, 2006, art. 1)

No obstante, al contrastar la formación en emprendimiento con los objetivos específicos de la formación para el emprendimiento (art. 12) se puede notar que, aunque la guía $n .^{\circ} 39$ (MEN, 2012) propone el desarrollo de competencias ciudadanas, en esta directiva ministerial se hace mayor hincapié en la generación de empresa y construcción de propuestas que contribuyan a la articulación con el sector productivo.

Otro referente que despertó la necesidad de abordar la temática del emprendimiento como cultura en la primera infancia es el vacío existente en la guía $n .^{\circ} 39$ cuando propone la cultura del emprendimiento como una "oportunidad para que el estudiante materialice sus actitudes emprendedoras y para la empresarialidad en el desarrollo de actividades y proyectos orientados a la creación de empresa con perspectiva de desarrollo sostenible con productividad económica, cultural, científica, tecnológica, deportiva o artística" (MEN, 2012, p.10). Así mismo, esta guía propone algunas estrategias para el fomento de la cultura del emprendimiento, pero solo desde el grado cuarto en adelante; aspecto que lleva a preguntarse ¿por qué la guía no plantea estrategias para los niveles de preescolar?, ¿será qué es difícil establecer estrategias para abordar la cultura del emprendimiento desde las competencias ciudadanas tal como lo propone la Ley 1014 del 2006?

De las tensiones referidas surgieron los siguientes objetivos: identificar las concepciones que sobre emprendimiento tienen las maestras de jardín y su puesta en escena de proyectos a partir de los conocimientos que ellas poseen del tema; y comprender la influencia que tiene la cultura del emprendimiento con relación a las competencias ciudadanas y el trabajo en equipo en niños y niñas del nivel jardín, a partir de las voces de las docentes.

\section{Marco conceptual}

Según los rastreos realizados sobre el tema de investigación, se encontraron algunos estudios de carácter local, nacional e internacional. Acá se mencionan algunos de los más relevantes como:

- Avance de competencias en desarrollo del emprendimiento infantil (Mateus y Galeano, 2012), realizado en el colegio Ciudad Patio Bonito, del sector sur de Bogotá en la localidad de Kennedy. El objetivo de este estudio fue incentivar las habilidades emprendedoras en niños desde los $7 \mathrm{o}$ 9 años hasta llegar a los 18. Se pudo observar 
la evolución de aspectos familiares y escolares que apuntan al desarrollo de habilidades como la seguridad al expresarse, tener autocontrol, ser líderes y pensar en soluciones. Como resultado de esta investigación se desarrolló una feria de innovación con miras al fortalecimiento de las habilidades empresariales.

- Concepción de la política en la formación para el emprendimiento en la infancia en cuatro instituciones educativas del área metropolitana del Valle de Aburrá. Una aproximación al análisis crítico del discurso (Varela y López, 2011), realizada en la Universidad San Buenaventura, en Medellín. Tuvo como objetivo conocer las opiniones que tenían los docentes acerca del concepto de emprendimiento en la formación de la primera infancia. Al mismo tiempo, buscó percibir las posibles razones que conllevan a la falta de implementación en sus aulas de clase. Los resultados arrojaron que los maestros no tienen fuentes confiables con las cuales puedan investigar sobre el emprendimiento, sumado al hecho de que en las instituciones privadas, de acuerdo con los participantes de la investigación, hay poco apoyo del Gobierno para la formación y actualización de sus maestros. También se encontró que a nivel profesional los docentes cuentan con poco tiempo para el desarrollo de las actividades y para la articulación con otras áreas. Por último, a pesar de que en la Ley 1014 de 2006 se habla de la obligación de fomentar en las IE la cultura del emprendimiento, no está muy interiorizada por los legisladores y actores educativos para dar a conocer nuevas posibilidades de aprendizajes significativos.

Dichas investigaciones fueron un foco fundamental para el desarrollo del proyecto "Cultura del emprendimiento en el contexto escolar", pues resaltan la importancia que tiene potenciar habilidades como la innovación, el liderazgo y el trabajo en equipo en la primera infancia. A su vez, permitieron ver el tema desde el punto de vista docente, como un guía que brinda espacios de aprendizajes a la hora de transmitir la cultura del emprendimiento.

En esta misma línea se le dio relevancia a cinco categorías de análisis que fueron: trabajo en equipo (Alcaraz, 2011), cultura (1987), emprendimiento, cultura del emprendimiento (Congreso de la República de Colombia, 2006; MEN, 2012). Y la quinta categoría, competencias ciudadanas (MEN, 2004), se toma como trasversal a las categorías enunciadas.

En este orden de ideas, en los estándares básicos de competencias ciudadanas, este último concepto se entiende como "el conjunto de conocimientos y de habilidades cognitivas, emocionales y comunicativas que, articulados entre sí, hacen posible que el ciudadano actúe de manera constructiva en la sociedad democrática" (MEN, 2004, p. 8). Al tiempo que desarrolla actitudes emprendedoras como "visión de futuro, pensamiento flexible, creatividad, manejo de herramientas tecnológicas, identificación de oportunidades y recursos en el entorno, innovación, materialización de ideas en proyecto, capacidad para asumir riesgos y comportamiento autorregulado" (MEN, 2004, p. 12). Estas habilidades y actitudes se concretan en la realidad a través del trabajo en equipo y de las diversas capacidades para ser y hacer con aquello que se tiene en un determinado tiempo y lugar.

Para Geertz (1987), la cultura es un sistema de significaciones que cobran vida a través de las diferentes representaciones que construyen los seres humanos gracias al lenguaje, las relaciones e interacciones que se tejen con otros seres humanos. Es así que la cultura en esta apuesta investigativa es esencial en tanto son las maestras quienes encarnan la palabra y desde esta generan en niños y niñas una cultura del emprendiendo.

Con respecto a las competencias ciudadanas y su relación con el emprendimiento como cultura, se puede subrayar que en el jardín de infantes los niños constantemente se ven enfrentados a diversas experiencias de aprendizaje que los confrontan. Es decir, en su proceso de socialización ellos siempre están en constante contacto con otros niños con los cuales, y gracias a las interacciones, desarrollan actitudes, valores, motivaciones (competencias ciudadanas), destrezas y conocimientos (competencias científicas) que les permiten aprehender, aprender y relacionarse con los otros. 
De acuerdo con lo anterior, lo que se espera con este artículo es dar a conocer la relación existente entre las concepciones de las muestras sobre la cultura del emprendimiento con las competencias ciudadanas y el trabajo en equipo en niños y niñas del nivel jardín de una IE que presta servicios educativos a la primera infancia.

\section{Metodología}

El proyecto denominado "Cultura del emprendimiento en el contexto escolar" se realizó en una IE que presta servicios de atención a la primera infancia; de carácter privado, cuya ubicación es la comuna 14 del área metropolitana, estrato socioeconómico 6, barrio Poblado. Este proyecto asumió el paradigma cualitativo desde un enfoque hermenéutico, basado en la estrategia de investigación del estudio de caso.

\section{Participantes del estudio}

Los participantes del estudio fueron cuatro maestras del nivel jardín, quienes debían ser: titulares de los niveles prejardín y jardín, licenciadas en Educación y con más de dos años en la IE. Por último, cabe resaltar que cada participante firmó el consentimiento informado para poder utilizar los datos que suministró a este proyecto; además, se les notificó que en ningún momento se revelaría sus identidades.

\section{Instrumentos}

Para la recolección de información se utilizaron cinco técnicas, las cuales fueron: el grupo focal para docentes; cuatro entrevistas semi-estructuradas (una a cada docente); ocho observaciones no participantes de las cuales fueron dos para cada docente; seis talleres lúdicos distribuidos en dos por semana con una duración de 30 minutos; y, por último, se utilizaron los diarios de campo para registrar la información de cada instrumento.

\section{Análisis}

Para el análisis de la información se tuvieron en cuenta dos matrices categoriales. En la primera, seguido de sistematizar y limpiar la información, se procedió a categorizar por colores, estos colores respondían a las categorías del proyecto. Luego se juntó las informaciones aportados por los participantes según los colores en los cuales se habían clasificado. Y en la segunda matriz se registraron las categorías y subcategorías definitivas con los respectivos autores que soportarían la discusión de los resultados (tabla 1 ).

Tabla 1. Matriz categorial

\begin{tabular}{|c|c|c|c|c|c|}
\hline Ámbito temático & Objetivos generales & Objetivos específicos & Categorías & Subcategorías & Autor \\
\hline \multirow{5}{*}{$\begin{array}{l}\text { Cultura del } \\
\text { emprendimiento } \\
\text { en el contexto } \\
\text { escolar }\end{array}$} & \multirow{5}{*}{$\begin{array}{l}\text { Comprender la } \\
\text { influencia que } \\
\text { tiene la cultura del } \\
\text { emprendimiento } \\
\text { en relación con } \\
\text { la creatividad y el } \\
\text { trabajo en equi- } \\
\text { po en los niños } \\
\text { y niñas del nivel } \\
\text { jardín de la guarde- } \\
\text { ría - preescolar } \\
\text { Cascanueces. }\end{array}$} & \multirow{5}{*}{$\begin{array}{l}\text { 1. Identificar los } \\
\text { aportes de la cultura } \\
\text { del emprendimiento } \\
\text { en el desarrollo de la } \\
\text { creatividad. } \\
\text { 2. Reconocer los apor- } \\
\text { tes que ha dejado la } \\
\text { cultura del emprendi- } \\
\text { miento en relación con } \\
\text { el trabajo en equipo. } \\
\text { 3. Identificar las } \\
\text { estrategias que utili- } \\
\text { zan los docentes para } \\
\text { fomentar la cultura del } \\
\text { emprendimiento en la } \\
\text { guardería-preescolar } \\
\text { Cascanueces, en el } \\
\text { nivel jardín. }\end{array}$} & -Cultura & $\begin{array}{l}\text {-Identidad } \\
\text {-Costumbres } \\
\text {-Sociedad }\end{array}$ & $\begin{array}{l}\text { Clifford Geertz } \\
(1987)\end{array}$ \\
\hline & & & -Emprendimiento & $\begin{array}{l}\text {-Economía } \\
\text {-Empresarialidad } \\
\text {-Liderazgo }\end{array}$ & $\begin{array}{l}\text { Ley } 1014 \text { de } 2006 \text {, } \\
\text { art. } 1\end{array}$ \\
\hline & & & $\begin{array}{l}\text {-Cultura del em- } \\
\text { prendimiento }\end{array}$ & $\begin{array}{l}\text {-Autonomía } \\
\text {-Resolución de } \\
\text { problemas } \\
\text {-Desafíos }\end{array}$ & $\begin{array}{l}\text { Cartilla guía n. }{ }^{\circ} 39 \\
(\text { MEN, 2012) }\end{array}$ \\
\hline & & & $\begin{array}{l}\text {-Trabajo en equipo } \\
\text {-Competencias } \\
\text { ciudadanas }\end{array}$ & $\begin{array}{l}\text {-Cooperación } \\
\text {-Comunicación } \\
\text {-Capacidad de } \\
\text { decisión }\end{array}$ & $\begin{array}{l}\text { Rafael Alcaraz } \\
(2011)\end{array}$ \\
\hline & & & Creatividad & $\begin{array}{l}\text {-Iniciativa } \\
\text {-Innovación } \\
\text {-Crear }\end{array}$ & De Bono (1994) \\
\hline
\end{tabular}

Fuente: elaboración propia de las autoras. 


\section{Resultados y discusión}

Concepciones de las maestras sobre los términos emprendimiento y cultura del emprendimiento

La cultura del emprendimiento desde el ámbito educativo va más allá de la creación de empresa. Está enfocado en la formación de seres integrales con proyectos de vida que aporten al desarrollo social; seres capaces de enfrentarse a desafíos en el mundo laboral, educativo y social. A su vez, el emprendimiento es definido como:

Una forma de pensar, razonar y actuar centrada en las oportunidades, planteada con visión global y Ilevada a cabo mediante un liderazgo equilibrado y la gestión de un riesgo calculado, su resultado es la creación de valor que beneficia a la empresa, la economía y la sociedad. (Congreso de la República de Colombia, 2006, art. 1)

Lo anterior permite inferir que el emprendimiento está orientado hacia una mirada empresarial para la adquisición de riquezas. Ambos conceptos están articulados en los proyectos pedagógicos, los cuales son obligatorios en las IE, en tanto que lo que se pretenden es el desarrollo de seres íntegros, la formación ciudadana en competencias laborales, posibilidades de autoempleo y trabajo en equipo en todos los miembros de la sociedad. No obstante, al indagarse con las maestras sobre sus claridades conceptuales y procedimentales en relación con la cultura del emprendimiento se pudo inferir que aunque las maestras no logran definir conceptualmente la cultura del emprendimiento, desde referentes legales sí tienen idea sobre el significado del mismo. Este asunto se puede notar en el relato de la maestra Margarita Ortiz ${ }^{6}$ cuando afirma:

Para mí emprendimiento es emprender, es iniciar algo que no está pero que nos va a aportar para la vida cotidiana y que sea en pro de nuestro bienestar y el de los demás. (Entrevista realizada el 10 de marzo de 2016)

Se utilizarán seudónimos con el fin de salvaguardar la identidad de los participantes en dicha investigación.
Según lo expresado, se infiere que aunque ella no logra conceptualizar en su discurso el término cultura del emprendimiento, sí se logra ver la forma como concibe el término cultura. Lo anterior se corrobora con lo expresado en la Ley 1014, la cual señala que esta es el conjunto de "valores, creencias, ideologías, hábitos, costumbres y normas, que comparten los individuos en la organización y que surgen de la interrelación social, los cuales generan patrones de comportamiento colectivos que establece una identidad entre sus miembros" (Congreso de la República de Colombia, 2006, p. 1); pero que, además, permite que estos se identifiquen con la organización. Al revisarse la misma ley se encuentra que el concepto emprendimiento se entiende como:

Una manera de pensar y actuar orientada hacia la creación de riqueza. Es una forma de pensar, razonar y actuar centrada en las oportunidades, planteada con visión global y llevada a cabo mediante un liderazgo equilibrado y la gestión de un riesgo calculado, su resultado es la creación de valor que beneficia a la empresa, la economía y la sociedad. (Congreso de la República de Colombia, 2006, p. 1)

Según esto, aunque la Ley es explicita señalando que las IE son las Ilamadas a potenciar el emprendimiento como cultura desde los primeros niveles y grados escolares. Además, aunque estas pretensiones para estos niveles se deben enfocar desde la articulación con las competencias ciudadanas, también es cierto que existen contradicciones en tanto que la cita anterior le apuesta al emprendimiento desde la creación y generación de empresa y el desarrollo de competencias para la productividad económica. Lo anterior, entonces, permite deducir que la poca claridad en los referentes legales Ileva a que las IE, y por supuesto los maestros, traten de articular estos mandatos desde lo que ellos creen que son los proyectos de emprendiendo. Veamos un relato de una maestra al respecto:

Para mí la palabra emprendimiento significa ser creativo, encontrar posibilidades ante algo que se le presente al niño, no necesariamente significa el que gane el dinero como empresario, sino que él es capaz 
de encontrar muchas posibilidades frente a lo que se le presente. (Entrevista realizada a Socorro Guzmán, 10 de marzo de 2016)

Del relato de la participante se infiere que, aunque ella no tiene claro el concepto de emprendimiento desde la Ley, sí intenta desarrollar habilidades y competencias en los niños como, por ejemplo, la capacidad para verbalizar lo que piensa, siente y desea realizar. Así mismo, en la voz de la maestra se puede reconocer que los proyectos de cultura del emprendimiento para la primera infancia deben sacar de los niños el potencial creativo e imaginativo a partir de la activación de la palabra, la cual emerge a través de la pregunta como posibilidad pedagógica en el aula. Este aspecto se evidenció en los relatos expresado por los niños cuando, a partir de una actividad propuesta por la maestra, uno señaló:

Profe, por qué mejor no hacemos una galería, que es donde venden obras hechas por otras personas. (Observación no participante realizada, Matías Agudelo del nivel jardín, 10 de marzo de 2016)

Este testimonio muestra que los niños, desde su experiencia contextual y vivencial, generan ideas para el desarrollo de los proyectos escolares; para este caso lo que expresa el niño es una idea de negocio tal cual como lo expresa la guía $n .^{\circ} 39$ del MEN. En este sentido es necesario que las prácticas educativas estén marcadas por "ambientes de aprendizaje muy significativos (que favorezcan) el desarrollo de todas las dimensiones" (Quintero, GaIlego, Ramírez y Jaramillo 2016, p. 27); pues si hay ambientes de aprendizaje favorables para el aprendizaje los niños y las niñas se generaran nuevas ideas y harán propuestas según sus intereses.

Ahora bien, cuando un niño propone en el aula de clase se da la oportunidad para que se abra un abanico de ideas para la interiorización del proyecto y esto apunta a una parte esencial de la cultura de emprendimiento en la cual se logra un aprendizaje bidireccional entre el estudiante y el docente. Conforme a lo anterior, se expone lo mencionado por la docente Lucía Quintero:
En el proyecto "la tienda" vamos a realizar una galería, se va a vender cuadros hechos por los niños. Esa idea surgió de ellos y se les planteaban preguntas como: ¿qué es una galería? La galería para nosotros es un término grandecito, yo pienso que para un niño simplemente van a ser cuadros, pero un niño me respondió: "profe en una galería vamos a ver una diversidad de pinturas", la palabra diversidad, uno cree que un niño no la va a emplear. Entonces, ellos mismos dieron la pauta para utilizar esa estrategia como actividad. (Entrevista realizada el 10 marzo del 2016)

En sintonía con lo enunciado hasta aquí, se hace necesario pensar que el emprendimiento como cultura es posible en el aula con los niños de primera infancia a partir de la recuperación de sus voces, sus intereses e inquietudes. Así lo afirma Gallego-Henao cuando señala "el reconocimiento y el respeto por los derechos de los niños y las niñas - especialmente el derecho a participar-, representan oportunidades para que estos mejoren sus condiciones de vida" (2015, p. 153). En cualquier proyecto de aula, y especialmente en el de emprendimiento, es necesario dar el protagonismo a los niños porque este tipo de proyectos no pueden estar desarticulados de sus experiencias de vida, ya que cuando el conocimiento está vinculado a la cotidianidad de los niños las ideas fluyen, la creatividad e innovación aflora y los procesos educativos se dinamizan. Al respecto, afirma una maestra:

La cultura del emprendimiento es más que todo tener una mente innovadora y creadora, no para recolectar plata, si no para hacer cosas diferentes con lo que tenemos en la institución. Por ejemplo, con los productos que nos da la granja o con el reciclaje tratamos que los niños innoven, sean creativos y hagan propuestas de cómo utilizar estos productos. Siempre buscamos mostrarles que el emprendimiento no es adquirir dinero, sino que también es ser creativos, es transformar lo que tenemos en otras cosas que nos pueden servir y que relacionan con sus contextos cercanos. Para ello, les hago preguntas sobre cómo podemos utilizar los recursos que tenemos. (Entrevista realizada a Sandra Restrepo, 10 de marzo de 2016). 
Lo mencionado por la docente denota que la cultura del emprendimiento, para ella, es retomar los elementos del contexto escolar para reinventarlos y desde allí potenciar la creatividad de los niños y el desarrollo de competencias para resolver diversas situaciones problemáticas. En este sentido, se puede subrayar que la creatividad viene del ser y el hacer, dejar a un lado las instrucciones exactas sobre lo que se pretende realizar, lo que permite potenciar el pensamiento creador en los niños y las niñas a partir de dejarlos ser y teniendo en cuenta que lo que se haga en esta etapa de la vida puede repercutir más adelante de manera significativa. En este caso, la creatividad se ve como la iniciativa que está aportando el estudiante a las estrategias del docente.

Lo anterior significa que cultura del emprendimiento es una estrategia pedagógica que depende en su totalidad de la manera como los maestros lo implementan en el aula de clase, a partir de las propuestas de los niños. Solo de esta manera se fortalecerá el avance en el desarrollo de competencias ciudadanas y laborales, en pos de lo que demanda el MEN con respecto a lo que se debe enseñar hoy sobre cultura del emprendimiento.

En suma, aunque las maestras no aluden al emprendimiento desde referentes teóricos, se pudo notar que sus proyectos sí se relacionan con el emprendimiento como cultura y que a través de estos se potencializan las competencias ciudadanas: cognitivas, emocionales, comunicativas e integradoras; así como las habilidades para la vida: el pensamiento creativo, la empatía, las relaciones interpersonales y el trabajo en equipo (Montoya, 2009).

\section{La cultura del emprendimiento: una oportunidad para potenciar el trabajo en equipo}

Cuando se habla de trabajo en equipo se debe hablar, en consecuencia, de una actitud cooperadora que orienta a una multitud a reunirse entorno a objetivos comunes y labrar para alcanzarlos. Incluso así, este concepto no se debe confundir con trabajo en grupo, porque trabajar en equipo implica un funcionamiento de alto nivel con el fin de que sus integrantes trabajen unidos. Al respecto, afirma Alcaraz:
El trabajo en equipo es un grupo de personas con una perspectiva organizada y clara sobre sus metas; que trabaja de manera independiente, en efecto, todos los miembros participan de forma activa y coordinada, aportan sus cualidades y buscan el mejor desempeño posible para el bien del proyecto y el cumplimiento de sus metas. (2011, p. 18)

Esta afirmación se materializó en una de los talleres llevados a cabo en el marco del trabajo de campo, cuando en una actividad denominada "La pirata Kate" los niños demostraron competencias ciudadanas tales como la escucha activa, la solución de situaciones problemáticas, la proposición de ideas para resolver problemas, el manejo de las emociones $y$, por supuesto, las competencias cognitivas. A continuación, se enuncia un fragmento del diario de campo:

[...] La pirata Kate dramatizó la historia y contó su tragedia del tesoro robado y escondido por Garfio, para que así los niños y las niñas se motivaran y le ayudaran a encontrar su tesoro; en este momento se mostraron animados y entusiasmados proponiendo ideas para atrapar al capitán Garfio. Por ejemplo, un niño dijo: "para encontrar el tesoro tenemos que mirar todas las casas, pongámosle trampas al capitán Garfio para poderlo atrapar, pero tienen que ser muy silenciosas para que el capitán no se dé cuenta y podamos atraparlo"; otro de los estudiantes refutó: "tenemos que trabajar todos en silencio y saber qué vamos a hacer primero por si se sale por el techo o por la puerta de atrás". Posteriormente, los niños salieron del salón persiguiendo a la pirata Kate en busca del tesoro perdido, al llegar al lugar se encontraron una nota del capitán Garfio la cual decía: "para poder encontrar el tesoro perdido debían atarse los pies con una banda elástica por parejas y trabajar juntos". Los niños de inmediato aceptaron la condición y se pusieron en actitud de búsqueda y al tiempo manifestaban algunas estrategias para poder cumplir el objetivo; por ejemplo, Santiago Rúa dijo: "tenemos que caminar con el mismo pie para no caernos, no corras porque me haces caer". Otra niña dijo: "contemos para dar el paso, no te corras mucho porque me jalas y no llegamos a ninguna parte". (Observación del 
taller n. ${ }^{\circ}$, realizado con los estudiantes de jardín el 11 de marzo de 2016)

De lo anterior, se resalta que uno de los momentos más relevantes surgió cuando debían atarse los pies en pareja para cumplir la misión del tesoro escondido. Se observó que los niños resolvieron la situación a través del diálogo, la escucha, los acuerdos y la toma de decisiones consensuada. Este tipo de competencias permitió vislumbrar que cuando un equipo se traza metas alcanzar el objetivo es fácil.

En sintonía con lo dicho, la cultura del emprendimiento aporta a la comprensión y reconocimiento de la diversidad, así como a la toma de conciencia por parte de los niños. Es decir, actividades que parecen tan simples y comunes les permite a ellos comprender que no son seres autosuficientes sino que necesitan de las otras personas para la subsistencia, el aprendizaje, la creación o la reinvención de las diversas situaciones. Es así, que se puede señalar que los proyectos de emprendimiento en el escenario escolar se convierten en una excusa para potenciar saber-es y hacer-es en contexto, así como las competencias ciudadanas.

No obstante, en el acompañamiento escolar no se puede olvidar que el trabajo con los niños inicia en el reconocimiento de las individualidades para luego generar un trabajo con el otro. Al respecto, una docente resalta:

Para mí, la cultura del emprendimiento aporta de manera directa al trabajo en equipo porque enseña a valorar el trabajo del otro. Que si nos comemos una fruta o una verdura, no es que voy a un supermercado y la compro, sino que hay personas que trabajan la tierra, que siembran el producto, que con eso sobrevive la gente del campo, que los niños vean que la gente del campo es la que siembra los alimentos y ya después se los vende a otras personas, la otra persona es la que lo lleva al supermercado. Yo, sobre todo, hago énfasis en esos aspectos. (Entrevista realizada a Sandra Restrepo, 10 de marzo de 2016)

Lo que la maestra deja ver en su discurso tiene directa relación con el trabajo en equipo. Se observa que este es fomentado de manera gradual a través de la cultura del emprendimiento; es decir, la docente enseña a los niños no solo emprendimiento sino que toma como excusa las actividades del proyecto de aula para articular competencias ciudadanas a las diferentes áreas. Esta articulación permite, a su vez, crear conciencia sobre el respeto y valoración del trabajo del otro, el cuidado del ambiente y el origen de los productos, entre otras cosas. Otra maestra subraya:

El trabajo en equipo es aprender a aceptar al otro, esperar el turno, socializar con el otro y saber que no solo soy yo, sino que también se debe compartir. Porque los niños están acostumbrados a que todo gira alrededor de ellos, especialmente en su casa y contexto familiar. (Entrevista realizada a Mónica Zuleta, 10 de marzo 2016)

Pero es en el ámbito escolar en el cual aprenden que el otro es necesario e importante para el desarrollo individual, social y personal. De ahí la responsabilidad social tan importante que tienen las maestras de primera infancia. Ellas, como las agentes educadoras secundarias, están Ilamadas a formar en y para la vida, así como entender que los proyectos son trasversales entre ellos y con las áreas del desarrollo infantil. En síntesis, cualquier tipo de proyecto que se trabaje con la primera infancia es susceptible de articularse con otras áreas del saber en tanto que quien imprime la intencionalidad es la maestra, y el niño desde sus saberes participa en estas propuestas haciendo parte, de tal manera que despliega habilidades y competencias que le servirán para su desarrollo humano y el encuentro e interacción con otros.

\section{Conclusiones}

Se pueden destacar ciertos puntos fundamentales que permiten comprender de manera más clara la influencia que tiene la cultura del emprendimiento en el desarrollo de habilidades como el trabajo en equipo y competencias ciudadanas en los niños y las niñas de la IE escenario del proyecto de investigación. Es así como, a través del emprendimiento como cultura, se logra potenciar la 
resolución de situaciones problemáticas, el razonamiento lógico, la capacidad de argumentación y capacidad de análisis y proposición. Así mismo, se potencializa una actitud cooperativa en la que se comprende que el otro también es importante y tiene sus propios puntos de vista, lo que por antonomasia mejora los procesos de convivencia no solo en el aula.

En esta misma línea, y de acuerdo con lo expuesto por los participantes de esta investigación, la cultura del emprendimiento tiene como ramificación esencial el trabajo en equipo, el cual se define desde los relatos como un proceso continuo que se fortalece a partir de los primeros años de vida. Para esto es necesario implementar estrategias pedagógicas que permitan el diálogo constante y actitudes cooperadoras de forma activa entre los estudiantes y que a su vez conlleva a pensar en el otro y brinda la oportunidad de crear aprendizajes colectivos.

Por ende, el trabajo en equipo puede ser afectado de forma positiva o negativa por el contexto familiar y educativo al que el niño o la niña están inmersos, porque allí la atención está centrada en ellos, lo que en ocasiones les dificulta pensar en el otro. Este aspecto conlleva a invitar a padres, maestros y cuidadores de la primera infancia para que repiensen sus prácticas educativas y de crianza, lo anterior en tanto que en ocasiones se les envía un mensaje equivocado a los niños sobre su lugar en el mundo. Es decir, es necesario permitirles que generen procesos empáticos de tal manera que sientan que no son los únicos en el mundo, sino que hay otros que merecen el mismo cuidado, respeto y atención que ellos.

Finalmente, otro de los componentes esenciales de la cultura del emprendimiento es el desarrollo de proyectos a partir de los gustos e intereses de los niños; proyectos que necesariamente deben estar articulados a experiencias y vivencias de estos, de tal manera que el aprendizaje sea significativo y contextualizado.

\section{Referencias}

Alcaraz, R. (2011). El emprendedor del éxito. México: McGraw Hill.

Congreso de la República de Colombia (2006). Ley 1014, de fomento a la cultura del emprendimiento. Recuperado de http://www.mineducacion. gov.co/1621/articles-94653_archivo_pdf.pdf

Gallego-Henao, A. M. (2015). Participación infantil... Historia de una relación de invisibilidad. Revista Latinoamericana de Ciencias Sociales, Niñez y Juventud, 13(1), 151-165. Recuperado de http:// www.scielo.org.co/pdf/rlcs/v13n1/v13n1a09.pdf

Geertz, C. (1987). La Interpretación de las culturas. Barcelona: Gedisa.

Mateus, C. y Galeano, P. (2012). Avance de competencias en desarrollo del emprendimiento infantil. Bogotá: Universidad Andina Simón Bolívar.

Ministerio de Educación Nacional (2004). Estándares básicos de competencias ciudadanas. Formar para la ciudadanía jsí es posible! Recuperado de https://www.mineducacion.gov. co/1621/articles-75768_archivo_pdf.pdf

Ministerio de Educación Nacional (2012). CartiIla guía $n .^{\circ}$ 39. Sobre la cultura del emprendimiento en los establecimientos educativos. Recuperado de http://www.mineducacion.gov. co/1759/articles-287822_archivo_pdf.pdf

Montoya, I. (2009). Habilidades para la vida. Revista de Formación del Profesorado, 4. Recuperado de http://cefire.edu.gva.es/sfp/revistacompartim/arts4/02_com_habilidades_vida.pdf

Quintero, S., Gallego, A., Ramírez, L. y Jaramillo, B. (2016). La formación integral de las maestras para la primera infancia: un reto inaplazable. Zona Próxima, 25, 22-33. DOI: https://doi. org/10.14482/zp.25.9788

Varela, R. y López, P. (2011). Concepción de la política en la formación para el emprendimiento en la infancia en cuatro instituciones educativas del área metropolitana de valle de Aburrá. Una aproximación al análisis crítico del discurso. Medellín, Colombia: Universidad de San Buenaventura, Facultad de Educación. 